\title{
Cavity-Enhanced Room-Temperature Magnetometry Using Absorption by Nitrogen-Vacancy Centers in Diamond
}

\author{
K. Jensen, ${ }^{1, *}$ N. Leefer ${ }^{1, \dagger}$ A. Jarmola, ${ }^{1}$ Y. Dumeige, ${ }^{2}$ V. M. Acosta, ${ }^{1}$ P. Kehayias, ${ }^{1}$ B. Patton, ${ }^{1,3}$ and D. Budker ${ }^{1, *}$ \\ ${ }^{1}$ Department of Physics, University of California, Berkeley, California 94720-7300, USA \\ ${ }^{2}$ Université de Rennes 1, CNRS, UMR 6082 FOTON, Enssat, 6 rue de Kerampont, CS 80518, 22305 Lannion cedex, France \\ ${ }^{3}$ Physik-Department, Technische Universität München, 85748 Garching, Germany
}

(Received 10 January 2014; published 23 April 2014)

\begin{abstract}
We demonstrate a cavity-enhanced room-temperature magnetic field sensor based on nitrogen-vacancy centers in diamond. Magnetic resonance is detected using absorption of light resonant with the $1042 \mathrm{~nm}$ spin-singlet transition. The diamond is placed in an external optical cavity to enhance the absorption, and significant absorption is observed even at room temperature. We demonstrate a magnetic field sensitivity of $2.5 \mathrm{nT} / \sqrt{\mathrm{Hz}}$, and project a photon shot-noise-limited sensitivity of $70 \mathrm{pT} / \sqrt{\mathrm{Hz}}$ for a few $\mathrm{mW}$ of infrared light, and a quantum projection-noise-limited sensitivity of $250 \mathrm{fT} / \sqrt{\mathrm{Hz}}$ for the sensing volume of $\sim 90 \mu \mathrm{m} \times 90 \mu \mathrm{m} \times 200 \mu \mathrm{m}$.

DOI: 10.1103/PhysRevLett.112.160802

PACS numbers: 07.55.Ge, 76.30.Mi, 81.05.ug
\end{abstract}

Optical sensing of magnetic fields [1], electric fields [2,3], rotations [4-6], and temperature [7-10] can be achieved using negatively charged nitrogen-vacancy (NV) centers in diamond. Single NV centers and ensembles of NV centers can be detected with high spatial resolution and can be used as sensors with $\mathrm{nm}, \mu \mathrm{m}$, or $\mathrm{mm}$ resolution [11-14]. Most of these sensors are based on fluorescence detection of the NV center's spin state and suffer from low photon detection efficiency and background fluorescence. Even with improved photon collection [15-17], current state-of-the-art magnetic field sensors [17] can only reach sensitivities which are several orders of magnitude worse than the quantum projection noise (PN) limited sensitivity [14,18] associated with the finite number of sensing spins. Sensors based on detection of infrared (IR) absorption [19] achieve high photon detection efficiency and can reach a sensitivity closer to the PN limit compared to sensors based on fluorescence detection when a cavity is used to enhance the detection contrast [20]. Previously, absorption of IR light has been used for magnetometry at cryogenic temperatures ( 70 K) [19] where the absorption is much stronger than at room temperature. In this work, a diamond plate is placed in an external cavity which enhances the absorption from the NV centers and the spin-state detection contrast. The device is used as a magnetic field sensor operated at room temperature, but could be used for other applications requiring high optical depth such as electromagnetically induced transparency [3]. Here the use of a cavity could enable low-power, high-fidelity nonlinear optical processes such as quantum optical storage [21].

The level structure of the NV center is shown in Fig. 1(a). Electron spin-triplet and spin-singlet states are labeled ${ }^{3} A_{2}$, ${ }^{3} E$ and ${ }^{1} E,{ }^{1} A_{1}$, respectively. The $\mathrm{NV}$ center can be excited optically from the ground state ${ }^{3} A_{2}$ to the state ${ }^{3} E$. From the ${ }^{3} E$ state, the NV center can decay to the ${ }^{3} A_{2}$ state through a spin-conserving transition which leads to fluorescence in the 637-800 nm wavelength range. It can also decay to the upper singlet state ${ }^{1} A_{1}$ through a spin-nonconserving transition, which occurs with higher probability for the $m_{s}= \pm 1$ states compared to the $m_{s}=0$ state $[22,23]$. From the ${ }^{1} A_{1}$ state, the NV center decays through a $1042 \mathrm{~nm}$ transition to the metastable ${ }^{1} E$ singlet state, which has a lifetime of $\sim 200 \mathrm{~ns}$ at room temperature [24]. The NV center then decays from the ${ }^{1} E$ state back to
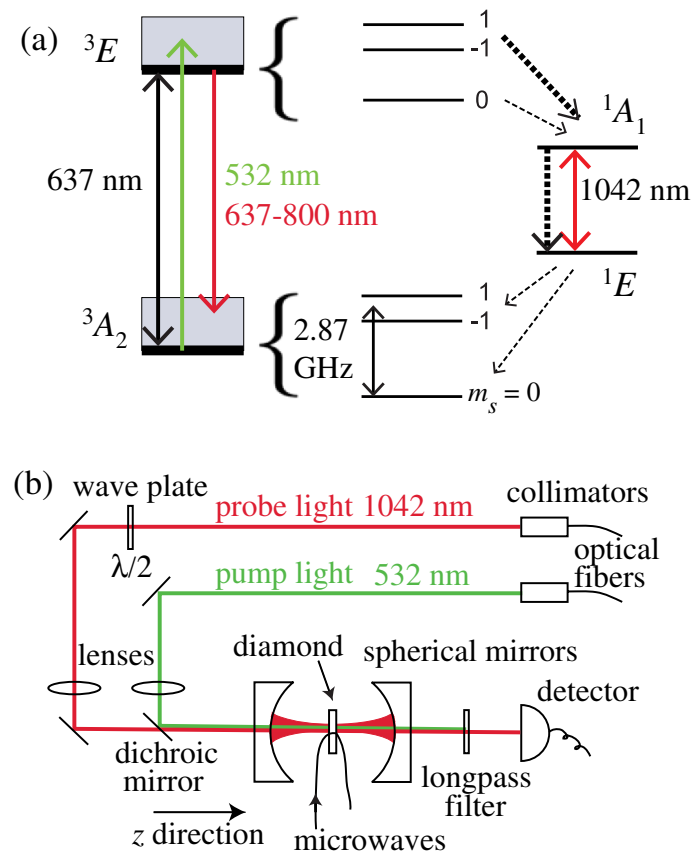

FIG. 1 (color online). (a) Levels and transitions of the NV center. Solid lines indicate optical and microwave transitions; dashed lines indicate nonradiative transitions. (b) Setup. The cavity was placed inside a sound insulation enclosure (not shown). 
the ${ }^{3} A_{2}$ ground state. Under continuous illumination with sufficiently strong green pump light at $532 \mathrm{~nm}$, the NV center is mainly in the ${ }^{3} A_{2} m_{s}=0$ ground state and the ${ }^{1} E$ metastable singlet state. Because of the spin-dependent transition rates, application of microwaves on resonance with the $m_{s}=0 \leftrightarrow m_{s}= \pm 1$ transitions leads to increased population of the ${ }^{1} E$ metastable singlet state. Absorption of $1042 \mathrm{~nm}$ light can therefore be used as a probe of the transitions within the spin-triplet ground state [25]

The experimental setup is shown in Fig. 1(b). $1042 \mathrm{~nm}$ light is provided by an external-cavity diode laser and $532 \mathrm{~nm}$ light is provided by a diode-pumped solid-state laser. The optical cavity consists of two spherical mirrors each with a radius of curvature of $r=5 \mathrm{~cm}$, an intensity reflectivity of $R=98(1) \%$ for $1042 \mathrm{~nm}$ light, and a transmittivity of $T=70(1) \%$ for $532 \mathrm{~nm}$ light. For the first measurements (shown in Fig. 2), we choose the mirror spacing $l=5.0 \mathrm{~cm}$ resulting in beam waist $w_{0}=91 \mu \mathrm{m}$ and Rayleigh range $z_{0}=2.5 \mathrm{~cm}$ for the $1042 \mathrm{~nm}$ TEM $_{00}$ cavity mode [26]. For the next measurements (shown in Fig. 3), we choose $l=2.5 \mathrm{~cm}$ resulting in $w_{0}=85 \mu \mathrm{m}$ and $z_{0}=2.2 \mathrm{~cm}$. The IR laser beam was matched to the $\mathrm{TEM}_{00}$ cavity mode in both cases. The pump laser beam was overlapped with the IR laser beam in the center of the cavity, but it was not mode matched to the cavity. The waist of the pump beam was 90(2) $\mu \mathrm{m}$ as measured with a CCD camera. Trace 1 in Fig. 2(a) shows the transmission of IR light through the cavity while scanning the laser frequency. The frequency interval between the two resonances is $c /(2 l)=3.0 \mathrm{GHz}$, where $l=5.0 \mathrm{~cm}$ and $c$ is the speed of light. From Lorentzian fits to the resonances in trace 1, we find the full width at half maximum of the resonances $\Delta \nu_{c}=14.9(15) \mathrm{MHz}$ and the cavity finesse $\mathscr{F}=[c /(2 l)] /$ $\Delta \nu_{c}=202(20)$. The measured finesse corresponds to mirror reflectivity $R=98.5(2) \%$ as calculated from Eq. (S3) in the Supplemental Material [27].
Diamond has a refractive index of $n=2.4$ which leads to a reflection of $17 \%$ for normal incidence at a diamond-air interface. Our diamond sample with size $3 \mathrm{~mm} \times 3 \mathrm{~mm} \times$ $0.2 \mathrm{~mm}$ is antireflection coated and has a measured total reflection from both sides of $0.1 \%$ at $1042 \mathrm{~nm}$. The sample was irradiated with relativistic electrons and annealed as in Ref. [14] in order to increase the density of NV centers. Trace 2 in Fig. 2(a) shows the transmission through the cavity when the diamond is inserted inside the cavity. The peak transmission decreases slightly compared to an empty cavity and the finesse is lowered to $\mathscr{F}=165(17)$ due to additional losses introduced by the diamond. This value corresponds to a single-pass loss in the diamond of $0.35 \%$, as calculated from Eq. (S3) with $R=98.5 \%$. The cavity resonance frequencies depend on the index of refraction of the diamond. We observe significant linear birefringence of the diamond [27], as seen in Trace 3 in Fig. 2(a), where twice the number of resonances are present in the spectrum. The large linear birefringence constrains the polarization of the light transmitted through the cavity to be either along or perpendicular to the diamond's axis of birefringence. In the measurements discussed below, we adjusted the input light polarization to maximize one cavity resonance [as in trace 2 in Fig. 2(a)] and locked the laser frequency to this resonance.

Absorption of light by an NV center depends on the light polarization, and for both the ${ }^{3} A_{2} \leftrightarrow{ }^{3} E$ triplet transition and the ${ }^{1} E \leftrightarrow{ }^{1} A_{1}$ singlet transition, the absorption is maximal when the polarization is perpendicular to the $\mathrm{NV}$ axis and zero when the polarization is along the NV axis [24]. The $\mathrm{NV}$ center's axis [28] can be aligned in four different ways in the diamond crystal, corresponding to the four [111] crystallographic directions. Our diamond is cut along one of the (100) planes and positioned such that its [100] axis coincide with the light propagation direction $[z$ direction in Fig. 1(b)]. In this case, the total absorption by all NV centers is relatively insensitive to the polarization of the
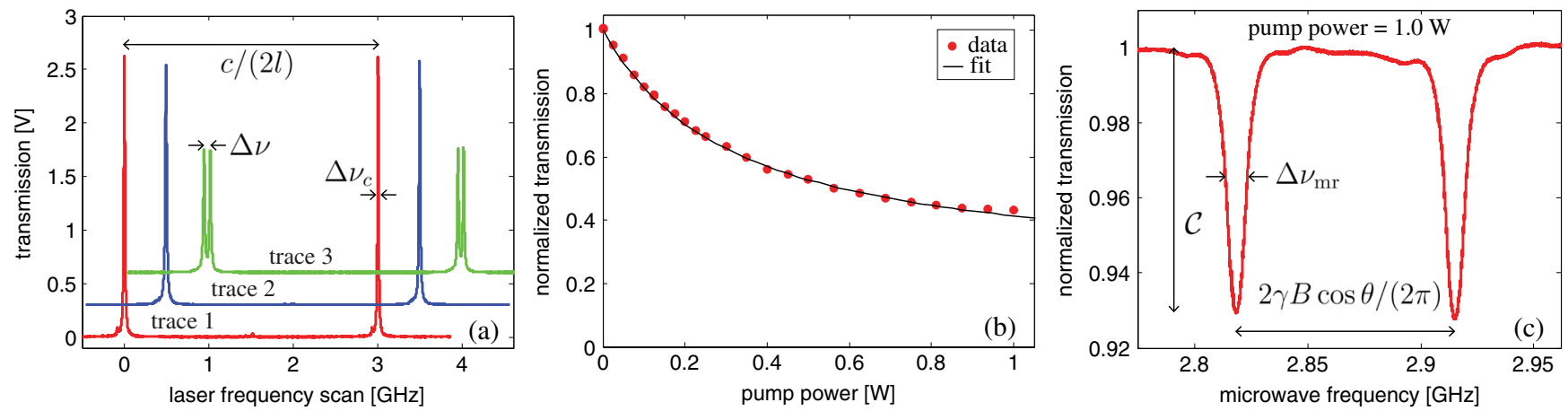

FIG. 2 (color online). (a) Transmission of IR light through the cavity when the IR laser frequency is scanned. Trace 1: the cavity is empty. Trace 2 and 3: The diamond is located inside the cavity. The experimental settings for trace 2 and 3 are the same except for the input light polarization. The spectra have been offset and shifted for clarity. The pump light was blocked for these measurements. (b) Transmission of IR light (with the laser locked to a cavity resonance) as a function of pump power measured before the cavity. The pump power inside the diamond is lower due to reflections from the front mirror of the cavity and the diamond surface. The transmission is here normalized to unity for zero pump power. (c) Transmission (normalized to unity off resonance) as a function of microwave frequency. A 2.99(1) mT magnetic field is aligned along the $z$ direction. 
pump and probe light. This is an advantage when the diamond is birefringent and the probe polarization cannot be chosen freely.

Figure 2(b) shows the cavity transmission of $1042 \mathrm{~nm}$ light as a function of $532 \mathrm{~nm}$ pump light power. The drop in transmission at higher pump powers is attributed to absorption from a larger number of NV centers in the singlet states. We observe a large $(>50 \%)$ change in transmission for high pump powers. Such a large change is possible at room temperature because the absorption is enhanced by the factor $2 \mathscr{F} / \pi$ due to the cavity compared to a single-pass scheme [27]. Absorption of IR light due to NV centers can be modeled as a pump power-dependent loss inside the cavity. The single-pass transmission through the diamond is $L_{0}-A_{0} P /\left(P+P_{\text {sat }}\right)$, where $L_{0}$ is the transmission coefficient in the absence of pump light, $P$ is the pump power, $P_{\text {sat }}$ is a saturation pump power, and $P /\left(P+P_{\text {sat }}\right)$ is the fraction of NV centers which are pumped into the metastable singlet state. The data in Fig. 2(b) are fitted to such a model [using Eq. (S2)] and we find $A_{0}=2.2(1) \%$ and $P_{\text {sat }}=0.88(3) \mathrm{W}$. The parameter $A_{0}$ is related to the density of $\mathrm{NV}$ centers through $n_{\mathrm{NV}} \approx A_{0} /\left(\sigma_{\mathrm{IR}} \times d\right)$ [27], where $\sigma_{\mathrm{IR}}=3_{-1}^{+3} \times 10^{-22} \mathrm{~m}^{2}$ is the cross section for IR light [20,33] and $d$ is the thickness of the diamond. We calculate $n_{\mathrm{NV}}=3.6(1.8) \times 10^{23} \mathrm{~m}^{-3}$ or, equivalently, 2(1) ppm which is in agreement with the expected value for this type of electron-irradiated diamond [14]. The saturation pump power $P_{\text {sat }}$ corresponds to a peak intensity $I_{0}=2 P_{\text {sat }} /\left(\pi w_{0}^{2}\right)=70 \mathrm{MW} / \mathrm{m}^{2}$, which is an order of magnitude smaller than the expected saturation intensity as calculated from the pump light cross section and the lifetime of the metastable singlet state [27]. The discrepancy could be due to other pump-power dependent effects such as photoionization of NV centers.

The NV center's ground-state energy levels shift due to the Zeeman effect in an applied magnetic field. The Zeeman effect can be described by the Hamiltonian $\mathscr{H}_{B}=\gamma \mathbf{B} \cdot \mathbf{S}$, where $\gamma=2 \pi \times 28.0 \mathrm{GHz} / \mathrm{T}$ is the gyromagnetic ratio for the NV center. A permanent ring magnet is used to apply a magnetic field, $\mathbf{B}=B \hat{\mathbf{z}}$, along the $z$ direction which coincides with a [100] direction. In this case, the angle between any NV center's axis and the magnetic field is $\theta=54.7^{\circ}$. For magnetic fields $B \ll 2 \pi D /(\gamma \cos \theta) \sim 0.2 \mathrm{~T}$, where $D=2.87 \mathrm{GHz}$ is the zero-field splitting of the NV center's ground state energy levels, the Zeeman shift depends to first order only on the projection of the magnetic field on the NV axis. The shift in $\mathrm{Hz}$ is $\approx m_{s} \gamma B \cos \theta /(2 \pi)$ and is the same for all NV centers, independent of their orientations. Microwaves are applied with a wire positioned on top of the diamond. Figure 2(c) shows the cavity transmission while the microwave frequency is scanned. The pump power was $1.0 \mathrm{~W}$ for this measurement. Two magnetic resonances are observed corresponding to the $m_{s}=0 \leftrightarrow m_{s}=-1$ and $m_{s}=0 \leftrightarrow m_{s}=+1$ transitions within the ground state. The resonance frequencies are $f_{\text {res }}=D \pm \gamma B \cos \theta /(2 \pi)$ and the magnetic field is determined to be 2.99(1) $\mathrm{mT}$ from the frequency difference between the resonances. The contrast $\mathscr{C}$ and the full width at half maximum $\Delta \nu_{\mathrm{mr}}$ of the magnetic resonances [see Fig. 2(c)] depend on the pump and microwave powers [34], which were chosen to optimize the magnetic field sensitivity. For the resonances in Fig. 2(c) we find $\mathscr{C}=7.1 \%$ and $\Delta \nu_{\mathrm{mr}}=9.0 \mathrm{MHz}$. When detecting IR absorption at room temperature, such a high contrast is only possible due to the cavity enhancement which increases $\mathscr{C}$ by the factor $2 \mathscr{F} / \pi \sim 75$. The sensitivity to magnetic fields aligned along the $z$ direction is in the absence of technical noise limited by the shot noise $(\mathrm{SN})$ of the probe light. For the spectrum presented in Fig. 2(c), we find a SN-limited sensitivity $\delta B_{\mathrm{SN}} \approx\left(2 \pi \Delta \nu_{\mathrm{mr}}\right) /(\gamma \mathscr{C} \sqrt{\mathscr{R}} \cos \theta)$ [3] of $70 \mathrm{pT} / \sqrt{\mathrm{Hz}}$ using a detected IR power of $2.3 \mathrm{~mW}$ ( $\mathscr{R}$ is the rate of detected photons).

In order to make a sensitive measurement of the magnetic field, we utilize a technique where the microwave frequency $f_{\mathrm{MW}}$ is modulated around a central frequency $f_{c}$ : $f_{\mathrm{MW}}=f_{c}+f_{\text {dev }} \cos \left(2 \pi f_{\text {mod }} t\right)$, where $f_{\text {mod }}$ is the modulation frequency and $f_{\text {dev }}$ is the amplitude of modulation. The detected signal (the transmission through the cavity) is demodulated with a lock-in amplifier referenced to the modulation frequency $f_{\text {mod }}$. The output of the lock-in amplifier is linear $S_{\mathrm{LI}} \approx \alpha\left(f_{c}-f_{\text {res }}\right)$ as a function of $f_{c}-f_{\text {res }}$ when $\left|f_{c}-f_{\text {res }}\right|<\Delta \nu_{\text {mr }} / 2$. Besides providing a linear magnetometer signal, the modulation technique reduces the sensitivity to technical low-frequency noise. Trace 1 in Fig. 3(a) shows the transmission while $f_{c}$ is scanned over the lowest-frequency resonance in Fig. 2(c). The fast modulation is not visible in this trace due to averaging. The slope $\alpha \propto \mathscr{C} / \Delta \nu_{\mathrm{mr}}$ is extracted from trace 2 in Fig. 3(a) which shows the output of the lock-in amplifier. Because of the finite response time of the NV centers to magnetic fields (see Fig. S1 [27]), the slope $\alpha$ also depends on $f_{\text {mod }}$. For $f_{\text {mod }}=15.8 \mathrm{kHz}, \alpha$ was reduced by a factor $\sim 2.2$ compared to low modulation frequencies. We chose $f_{\text {mod }}=15.8 \mathrm{kHz}$ since this maximized the measured signal-to-noise ratio. For $f_{\text {mod }}<10 \mathrm{kHz}$, intensity noise of the pump laser became a dominant noise source.

The lock-in signal is linear for small changes $\Delta B_{z}$ in the magnetic field component pointing along the bias field $B \hat{\mathbf{z}}$, when the central microwave frequency is set to $f_{c}=D-\gamma B \cos \theta /(2 \pi)$. Under this condition, the output of the lock-in amplifier was recorded for $10 \mathrm{~s}$. Knowing the slope $\alpha$, the change in magnetic field $\Delta B_{z}(t)=$ $-2 \pi S_{\mathrm{LI}}(t) /(\alpha \gamma \cos \theta)$ as a function of time can be calculated [see inset in Fig. 3(b)]. The root-mean-square (rms) magnetic field fluctuations [which equals the square-root of the power spectral density of $\left.\Delta B_{z}(t)\right]$ are shown in Fig. 3(b). For frequencies in the range $10-500 \mathrm{~Hz}$ we reach a noise floor of $2.7 \mathrm{nT} / \sqrt{\mathrm{Hz}}$. For frequencies below $10 \mathrm{~Hz}$ additional noise is present. Above $500 \mathrm{~Hz}$ the noise decreases due to the finite time constant of the lock-in amplifier (here $320 \mu \mathrm{s}$ ), which reduces the bandwidth to $\sim 1 \mathrm{kHz}$. Several noise peaks are also present. 

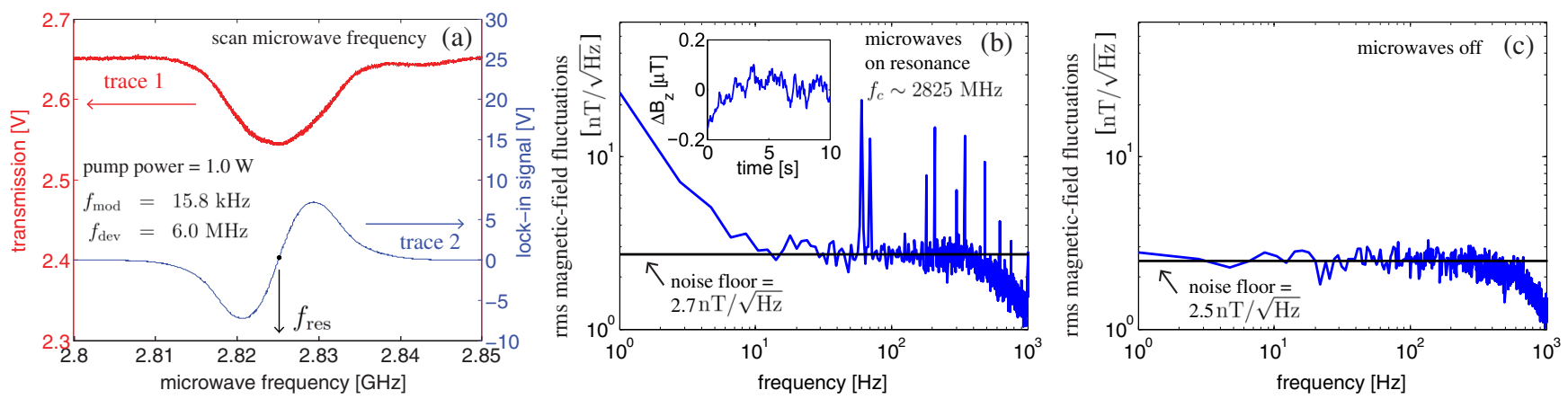

FIG. 3 (color online). (a) Cavity-transmission signal (top trace) and lock-in signal (bottom trace) as a function of the central microwave frequency $f_{c}$ which is scanned over a magnetic resonance. (b) Magnetic field noise spectrum. Inset: Magnetic field deviations from $B=2.5(2) \mathrm{mT}$ as a function of time. The data were binned in $0.05 \mathrm{~s}$ intervals for clarity. (c) Noise spectrum for the case where the microwaves were turned off.

The magnetic-resonance position depends on the temperature $\mathscr{T}$, since around room temperature the zero-field splitting $D$ depends linearly on $\mathscr{T}$ with the slope $d D / d \mathscr{T}=$ $-74 \mathrm{kHz} / \mathrm{K}$ [7]. The sensitivity of our magnetometer to temperature changes is $\gamma \cos \theta /(2 \pi d D / d \mathscr{T})=0.22 \mathrm{~K} / \mu \mathrm{T}$. Figure 3(c) shows the noise spectrum when the microwaves are off, where the absorption due to NV centers does not depend on magnetic field or temperature. In this case we do not observe the additional low-frequency noise or the noise peaks. Based on this observation and additional measurements performed with a commercial magnetometer (Honeywell HMC2003), we determine that the noise peaks are due to magnetic noise in the lab while the lowfrequency noise is due to temperature fluctuations of the diamond. The high pump power leads to significant heating of the diamond (around $40 \mathrm{~K}$ increase for $1.0 \mathrm{~W}$ pump power as measured from the shift in resonance frequency). Thermal fluctuations could arise if the diamond is not in thermal equilibrium or if the pump power fluctuates. The low-frequency noise could be reduced by improving the thermal contact of the diamond to the mount, by actively stabilizing the temperature of the diamond, or by reducing or stabilizing the pump power. Alternatively, temperature changes could be distinguished from magnetic field changes by monitoring the positions of both resonances in Fig. 2(c) or by using a quantum beat technique [35].

The noise floor in Fig. 3(c) is $2.5 \mathrm{nT} / \sqrt{\mathrm{Hz}}$ and is limited by laser frequency noise and/or vibrations of the cavity. The cavity length was $2.5 \mathrm{~cm}$ for the measurements presented in Fig. 3, and $5 \mathrm{~cm}$ for the measurements presented in Fig. 2. The cavity resonance width $\left[\Delta \nu_{c}\right.$ in Fig. 2(a)] is larger for the shorter cavity. A short cavity is preferable, since in this case the magnetometer will be less sensitive to laser frequency noise. We observed an improvement of the noise floor with the shorter cavity, demonstrating that laser frequency noise was limiting the sensitivity for the longer cavity. We believe that laser frequency noise still limits the sensitivity for the shorter cavity and that further reduction of the cavity length would be beneficial. The contribution from the probe laser intensity noise to the sensitivity is $\sim 0.8 \mathrm{nT} / \sqrt{\mathrm{Hz}}$ for $f_{\text {mod }}=15.8 \mathrm{kHz}$.

We have demonstrated a cavity-enhanced, roomtemperature magnetometer based on IR absorption using $\mathrm{NV}$ centers in diamond. We reach a noise floor of $2.5 \mathrm{nT} /$ $\sqrt{\mathrm{Hz}}$ and project a SN-limited sensitivity of $70 \mathrm{pT} / \sqrt{\mathrm{Hz}}$ for our current apparatus. These values can be compared to the results of 100 and $9 \mathrm{pT} / \sqrt{\mathrm{Hz}}$, respectively, measured in Ref. [17], which used a "side-collection" technique to improve low detection efficiency for fluorescent photons. Whereas Ref. [17] performed measurements of a highfrequency ac magnetic field, the present technique is sensitive to magnetic fields from dc-1 kHz. Possible applications of our magnetometer include mapping of magnetic fields and gradients inside magnetic enclosures, zero field nuclear magnetic resonance microfluidic detection [36], and detection of magnetic microparticles [37]. The SN-limited sensitivity of our device could be improved by increasing the probe power. The $1042 \mathrm{~nm}$ transition saturation intensity was predicted to be $\sim 500 \mathrm{GW} / \mathrm{m}^{2}$ [20], which for a $90 \mu \mathrm{m}$ beam waist corresponds to a saturation power of $6.4 \mathrm{~kW}$. Therefore, saturation effects will not be important. A realistic increase of the probe power by a factor of 100 would increase the SN-limited sensitivity by a factor of 10 to $\sim 7 \mathrm{pT} / \sqrt{\mathrm{Hz}}$. Detection of magnetic resonance using IR absorption enhanced by a cavity has the advantage that $100 \%$ contrast is possible using a critically coupled cavity [20], and this method is in principle more sensitive than methods based on detecting fluorescence [20]. For any detection method, the sensitivity is fundamentally limited by the quantum projection noise $[14,18]$ $\delta B_{\mathrm{PN}} \approx(2 \pi) /\left(\gamma \sqrt{N_{\mathrm{NV}} T_{2}}\right) \sim 250 \mathrm{fT} / \sqrt{\mathrm{Hz}}$, where $N_{\mathrm{NV}}$ is the number of $\mathrm{NV}$ centers and $T_{2}=1 /\left(\pi \Delta \nu_{\mathrm{mr}}\right)$ is the coherence time. A sensitivity in this range is feasible using a critically coupled cavity and an IR power of a few hundreds of $\mathrm{mW}$. Optimizing the diamond sample in terms of density of NV centers, substitutional nitrogen atoms, and ${ }^{13} \mathrm{C}$ nuclei could, together with dynamical decoupling sequences [1,38], lead to narrower magnetic resonances and even higher sensitivity. 
This research was supported by the AFOSR/DARPA QuASAR program and by NSF through Grant No. ECCS1202258. K. J. was supported by the Danish Council for Independent Research I Natural Sciences and the Carlsberg Foundation. Y. D. acknowledges support from Institut Universitaire de France. We thank G. Vasilakis for commenting on the manuscript.

*kjensen@nbi.dk

†naleefer@uni-mainz.de

¥dbudker@gmail.com

[1] J. M. Taylor, P. Cappellaro, L. Childress, L. Jiang, D. Budker, P. R. Hemmer, A. Yacoby, R. Walsworth, and M. D. Lukin, Nat. Phys. 4, 810 (2008).

[2] F. Dolde, H. Fedder, M. W. Doherty, T. Nöbauer, F. Rempp, G. Balasubramanian, T. Wolf, F. Reinhard, L. C. L. Hollenberg, F. Jelezko, and J. Wrachtrup, Nat. Phys. 7, 459 (2011).

[3] V. M. Acosta, K. Jensen, C. Santori, D. Budker, and R. G. Beausoleil, Phys. Rev. Lett. 110, 213605 (2013).

[4] D. Maclaurin, M. W. Doherty, L. C. L. Hollenberg, and A. M. Martin, Phys. Rev. Lett. 108, 240403 (2012).

[5] M. P. Ledbetter, K. Jensen, R. Fischer, A. Jarmola, and D. Budker, Phys. Rev. A 86, 052116 (2012).

[6] A. Ajoy and P. Cappellaro, Phys. Rev. A 86, 062104 (2012).

[7] V. M. Acosta, E. Bauch, M. P. Ledbetter, A. Waxman, L.-S. Bouchard, and D. Budker, Phys. Rev. Lett. 104, 070801 (2010).

[8] G. Kucsko, P. C. Maurer, N. Y. Yao, M. Kubo, H. J. Noh, P. K. Lo, H. Park, and M. D. Lukin, Nature (London) 500, 54 (2013).

[9] D. M. Toyli, C. F. de las Casas, D. J. Christle, V. V. Dobrovitski, and D. D. Awschalom, Proc. Natl. Acad. Sci.U.S.A. 110, 8417 (2013).

[10] P. Neumann, I. Jakobi, F. Dolde, C. Burk, R. Reuter, G. Waldherr, J. Honert, T. Wolf, A. Brunner, J. H. Shim, D. Suter, H. Sumiya, J. Isoya, and J. Wrachtrup, Nano Lett. 13, 2738 (2013).

[11] G. Balasubramanian, I. Y. Chan, R. Kolesov, M. Al-Hmoud, J. Tisler, C. Shin, C. Kim, A. Wojcik, P. R. Hemmer, A. Krueger, T. Hanke, A. Leitenstorfer, R. Bratschitsch, F. Jelezko, and J. Wrachtrup, Nature (London) 455, 648 (2008).

[12] J. R. Maze, P. L. Stanwix, J. S. Hodges, S. Hong, J. M. Taylor, P. Cappellaro, L. Jiang, M. V. Gurudev Dutt, E. Togan, A. S. Zibrov, A. Yacoby, R. L. Walsworth, and M. D. Lukin, Nature (London) 455, 644 (2008).

[13] E. Rittweger, K. Young Han, S. E. Irvine, C. Eggeling, and S. W. Hell, Nat. Photonics 3, 144 (2009).

[14] V. M. Acosta, E. Bauch, M. P. Ledbetter, C. Santori, K.-M. C. Fu, P. E. Barclay, R. G. Beausoleil, H. Linget, J. F. Roch, F. Treussart, S. Chemerisov, W. Gawlik, and D. Budker, Phys. Rev. B 80, 115202 (2009).

[15] J. P. Hadden, J. P. Harrison, A. C. Stanley-Clarke, L. Marseglia, Y.-L. D. Ho, B. R. Patton, J. L. O'Brien, and J. G. Rarity, Appl. Phys. Lett. 97, 241901 (2010).
[16] P. Siyushev, F. Kaiser, V. Jacques, I. Gerhardt, S. Bischof, H. Fedder, J. Dodson, M. Markham, D. Twitchen, F. Jelezko, and J. Wrachtrup, Appl. Phys. Lett. 97, 241902 (2010).

[17] D. Le Sage, L. M. Pham, N. Bar-Gill, C. Belthangady, M. D. Lukin, A. Yacoby, and R. L. Walsworth, Phys. Rev. B 85, 121202 (2012).

[18] D. Budker and M. Romalis, Nat. Phys. 3, 227 (2007).

[19] V. M. Acosta, E. Bauch, A. Jarmola, L. J.Zipp, M. P. Ledbetter, and D. Budker, Appl. Phys. Lett. 97, 174104 (2010).

[20] Y. Dumeige, M. Chipaux, V. Jacques, F. Treussart, J.-F. Roch, T. Debuisschert, V. M. Acosta, A. Jarmola, K. Jensen, P. Kehayias, and D. Budker, Phys. Rev. B 87, 155202 (2013).

[21] K. Heshami, C. Santori, B. Khanaliloo, C. Healey, V. M. Acosta, P. E. Barclay, and C. Simon, arXiv:1312.5342v1.

[22] L. Robledo, H. Bernien, T. van der Sar, and R. Hanson, New J. Phys. 13, 025013 (2011).

[23] J.-P Tetienne, L. Rondin, P. Spinicelli, M. Chipaux, T. Debuisschert, J.-F Roch, and V. Jacques, New J. Phys. 14, 103033 (2012).

[24] V. M. Acosta, A. Jarmola, E. Bauch, and D. Budker, Phys. Rev. B 82, 201202 (2010).

[25] It is also possible to use transmission of green light as a probe of the spin-triplet ground state. However, very low contrasts of the magnetic resonances were observed with this detection method; see Erik Bauch, Diploma Thesis, Technische Universität Berlin, 2010.

[26] P. W. Milonni and J. H. Eberly, Lasers (Wiley, New York, 1988).

[27] See Supplemental Material at http://link.aps.org/ supplemental/10.1103/PhysRevLett.112.160802 which includes Refs. [29-32].

[28] The NV center's axis corresponds to the vector connecting the nitrogen atom and the neighboring vacancy.

[29] I. Friel, S. L. Clewes, H. K. Dhillon, N. Perkins, D. J. Twitchen, and G. A. Scarsbrook, Diam. Relat. Mater. 18, 808 (2009).

[30] A. Batalov, C. Zierl, T. Gaebel, P. Neumann, I.-Y. Chan, G. Balasubramanian, P. R. Hemmer, F. Jelezko, and J. Wrachtrup, Phys. Rev. Lett. 100, 077401 (2008).

[31] T.-L. Wee, Y.-K. Tzeng, C.-C. Han, H.-C. Chang, W. Fann, J.-H. Hsu, K.-M. Chen, and Y.-C. Yu, J. Phys. Chem. A 111, 9379 (2007).

[32] C. S. Shin, C. E. Avalos, M. C. Butler, D. R. Trease, S. J. Seltzer, J. P. Mustonen, D. J. Kennedy, V. M. Acosta, D. Budker, A. Pines, and V. S. Bajaj, J. Appl. Phys. 112, 124519 (2012).

[33] P. Kehayias, M. W. Doherty, D. English, R. Fischer, A. Jarmola, K. Jensen, N. Leefer, P. Hemmer, N. B. Manson, and D. Budker, Phys. Rev. B 88, 165202 (2013).

[34] K. Jensen, V. M. Acosta, A. Jarmola, and D. Budker, Phys. Rev. B 87, 014115 (2013).

[35] K. Fang, V. M. Acosta, C. Santori, Z. Huang, K. M. Itoh, H. Watanabe, S. Shikata, and R. G. Beausoleil, Phys. Rev. Lett. 110, 130802 (2013).

[36] M. P. Ledbetter, I. M. Savukov, D. Budker, V. Shah, S. Knappe, J. Kitching, D. J. Michalak, S. Xu, and A. Pines, Proc. Natl. Acad. Sci. U.S.A. 105, 2286 (2008).

[37] D. Maser, S. Pandey, H. Ring, M. P. Ledbetter, S. Knappe, J. Kitching, and D. Budker, Rev. Sci. Instrum. 82, 086112 (2011).

[38] N. Bar-Gill, L. M. Pham, A. Jarmola, D. Budker, and R. L. Walsworth, Nat. Commun. 4, 1743 (2013). 